\title{
Transport and Strong-Correlation Phenomena in Carbon Nanotube Quantum Dots in a Magnetic Field
}

\author{
M. Mizuno ${ }^{1}$, Eugene H. Kim ${ }^{1}$ and G. B. Martins ${ }^{2}$ \\ 1 Department of Physics, University of Windsor, Windsor, Ontario, Canada N9B 3P4 \\ 2 Department of Physics, Oakland University, Rochester, Michigan 48309
}

\begin{abstract}
Transport through carbon nanotube (CNT) quantum dots (QDs) in a magnetic field is discussed. The evolution of the system from the ultraviolet to the infrared is analyzed; the strongly correlated (SC) states arising in the infrared are investigated. Experimental consequences of the physics are presented - the SC states arising at various fillings are shown to be drastically different, with distinct signatures in the conductance and, in particular, the noise. Besides CNT QDs, our results are also relevant to double QD systems.
\end{abstract}

Since their discovery, carbon nanotubes (CNTs) have been the subject of intense activity; [1] in particular, experiments on transport in CNTs have revealed a wealth of exciting phenomena. Indeed, long metallic CNTs have been shown to behave as quantum wires; 2, 3] negative differential resistance has been observed in semiconducting CNTs. [4] Furthermore, short CNTs have been shown to behave as quantum dots (QDs), [3, [5, [6] exhibiting Coulomb blockade (CB) phenomenology [7] known from gated two-dimensional semiconducting structures.

QDs have spurred a renewed excitement about the Kondo effect (KE), as they allow detailed investigations of the phenomena. [8] In this regard, CNT QDs are ideal for studies of Kondo physics. Indeed, initial experiments displayed an $\mathrm{SU}(2) \mathrm{KE}$ arising from the electron's spin; $[\underline{6}]$ more recently, orbital[9] as well as SU(4) KEs have been observed. [9, 10, 11] Furthermore, CNT QDs afford the possibility of tuning between a variety of stronglycorrelated (SC) states with a magnetic field. [9, 10]

In this work, we consider transport through CNT QDs, focussing on their behavior in a magnetic field. We analyze the system's evolution from the ultraviolet (UV) to the infrared (IR) fixed points (FPs); we discuss the KEs that arise and their consequences. More specifically, we consider the KEs arising from a single electron (referred to as 1/4-filled) as well as two electrons (referred to as 1/2-filled) occupying the energy levels of the CNT QD closest to the Fermi energy $E_{F}$ of the leads. While previous works detailed the properties of the 1/4-filled QD, 12] we show that the KEs arising from the $1 / 4$-filled and $1 / 2$ filled QDs are drastically different; these differences have pronounced observable consequences.

In what follows, we will be interested in the system's low-energy physics; hence, we focus on the energy levels of the CNT QD closest to $E_{F}$ of the leads. In the absence of magnetic fields, there are two degenerate energy levels, [9, 13] which we label as $\alpha$ and $\beta$. The Hamiltonian we consider is

$$
\begin{gathered}
H_{\mathrm{QD}}=\frac{E_{C}}{2}\left(\hat{N}-N_{0}\right)^{2}-\frac{h_{0}}{2} \sum_{s}\left(\hat{n}_{\alpha s}-\hat{n}_{\beta s}\right) \\
+\sum_{\kappa, s}\left(\left[t_{1} \psi_{1 \kappa s}^{\dagger}(0)+t_{2} \psi_{2 \kappa s}^{\dagger}(0)\right] d_{\kappa s}+\text { h.c. }\right),
\end{gathered}
$$

where $\psi_{i \kappa s}^{\dagger}(0)$ creates an electron (at $\left.x=0\right)$ with spin- $s$ in band- $\kappa$ from lead- $i(i=1,2) ; d_{\kappa s}^{\dagger}$ creates an electron with spin-s in orbital- $\kappa(\kappa=\alpha, \beta)$ on the QD; $\hat{n}_{\kappa s}=d_{\kappa s}^{\dagger} d_{\kappa s}$ and $\hat{N}=\sum_{\kappa, s} \hat{n}_{\kappa s} ; N_{0}$ is the optimal number of electrons on the $\mathrm{QD}$, which can be controlled by a gate voltage; $E_{C}$ is the charging energy; $t_{i}$ is the tunneling matrix element between lead- $i$ and the QD; $h_{0}$ is a magnetic field. In this work, we take the $\left\{t_{i}\right\}$ to conserve the orbital quantum number (which is relevant to the experiments in Refs. 10 and 11); 14 as a result, the system has an SU(4) symmetry when $h_{0}=0$. [15] $h_{0}$, which would arise from a magnetic field applied parallel to the CNT's axis, splits the $\alpha$ and $\beta$ orbitals. Throughout this work, we employ units where $\hbar=1$.

It should be noted $h_{0}$ would also give rise to a Zeeman splitting, but this splitting is considerably smaller than the orbital splitting, particularly for larger diameter CNTs. Indeed, the orbital moment $\mu_{\text {orb }}$ of a $5 \mathrm{~nm}$ diameter CNT was found to be $\mu_{\text {orb }} \simeq 1.5 \mathrm{meV} / \mathrm{T}[13]$ i.e. $\mu_{\mathrm{orb}} \simeq 26 \mu_{B}$. [ $\mu_{B}$ is the Bohr magneton.] As we will be interested in small fields $-h_{0} \sim \mathcal{O}\left(T_{K}^{\mathrm{SU}(4)}\right)$, where $T_{K}^{\mathrm{SU}(4)}$ is given by Eq. [5 - the Zeeman splitting will have very small effects. Therefore, in what follows, we focus on the orbital splitting.

We begin our discussion of the properties of CNT QDs by considering the current $I=\langle\hat{I}\rangle$, where $\hat{I}$ is the current operator

$$
\hat{I}=-i e t_{1} \sum_{\kappa, s}\left[\psi_{1 \kappa s}^{\dagger}(0) d_{\kappa s}-d_{\kappa s}^{\dagger} \psi_{1 \kappa s}(0)\right]
$$

( $e$ is the electron's charge); in particular, we compute the conductance $G=d I / d V$ vs. $N_{0}$ (in linear response). We are interested in the behavior of $G$ as $h_{0}$ is varied, as well as how $G$ evolves (with temperature) from the UV to the IR FPs. To understand the IR behavior, $G$ was computed as per Ref. 16 using the logarithmic-descretization embedded cluster approximation (LDECA) 17] and the Friedel sum rule [18]; to treat the UV regime $-T \gg \Gamma_{i}$, where $\Gamma_{i}=2 \pi \rho_{0} t_{i}^{2}$ with $\rho_{0}$ being the electrons' density of states in the leads - we employed a master equation approach. 19.

Fig. 11 shows $G$ vs. $N_{0}$ in the UV regime for several values of $h_{0}$. Letting $\Gamma_{0}=2 \Gamma_{1} \Gamma_{2} /\left(\Gamma_{1}+\Gamma_{2}\right)$,

$$
\begin{aligned}
G & =e^{2} \Gamma_{0} \sum_{\left\{N_{\alpha}, N_{\beta}, N_{\alpha}^{\prime}, N_{\beta}^{\prime}\right\}} \max \left\{M_{N_{\alpha} N_{\beta}}, M_{N_{\alpha}^{\prime} N_{\beta}^{\prime}}\right\} P_{N_{\alpha} N_{\beta}} \\
& \times \frac{\exp \left[\left(E_{N_{\alpha} N_{\beta}}-E_{N_{\alpha}^{\prime} N_{\beta}^{\prime}}\right) / T\right]+1}{8 T \cosh ^{2}\left[\left(E_{N_{\alpha} N_{\beta}}-E_{N_{\alpha}^{\prime} N_{\beta}^{\prime}}\right) / 2 T\right]}
\end{aligned}
$$




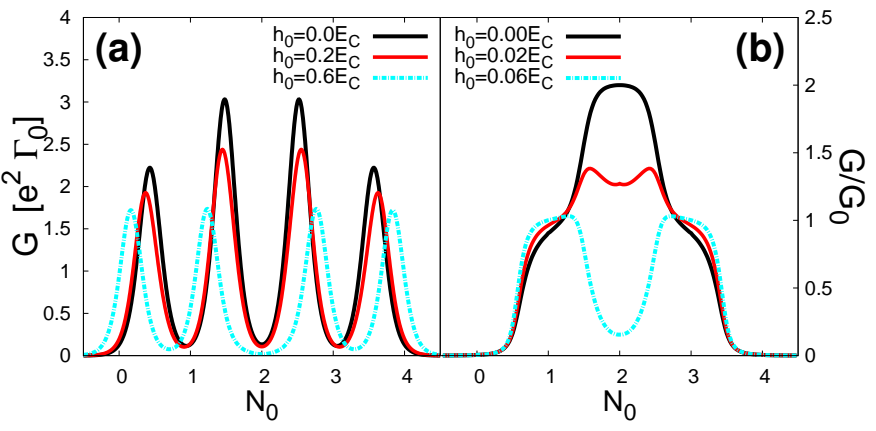

FIG. 1: $G=d I / d V$ vs. $N_{0}$ in linear response for several values of $h_{0}$ : (a) $T=0.1 E_{C}$ and (b) $T=0$.

where $P_{N_{\alpha} N_{\beta}}$ is the probability for the QD to be in a state with $N_{\alpha}\left(N_{\beta}\right)$ electrons in the $\alpha(\beta)$ orbital, $E_{N_{\alpha} N_{\beta}}$ is the energy of the state with $M_{N_{\alpha} N_{\beta}}$ being the number of these states, and the $\left\{N_{\alpha}, N_{\beta}, N_{\alpha}^{\prime}, N_{\beta}^{\prime}\right\}$ satisfy $\left(N_{\alpha}+N_{\beta}\right)-\left(N_{\alpha}^{\prime}+N_{\beta}^{\prime}\right)=1$. In Fig. 1a, we observe the well-known CB peaks for $N_{0}=N+1 / 2(N$ is an integer $)$ and valleys for $N_{0}=N$. When $h_{0}=0$, the system has an $\mathrm{SU}(4)$ symmetry; the two middle peaks have more spectral weight e.g. the peak at $N_{0}=3 / 2$ (due to fluctuations between states with $N=1$ and $N=2$ ) has more spectral weight than the peak at $N_{0}=1 / 2$ (due to fluctuations between states with $N=0$ and $N=1$ ). From the above expression for $G$, this occurs because there are more states with $N=2$ than $N=1$ or $N=0$. When $h_{0} \neq 0$, the $\mathrm{SU}(4)$ symmetry is reduced to $\mathrm{SU}(2)$; as a result, the peaks are split and the spectral weight becomes evenly distributed.

Fig. $1 \mathrm{~b}$ shows $G / G_{0}$ vs. $N_{0}$ at $T=0$, where $G_{0}=\left(e^{2} / \pi\right) 4 \Gamma_{1} \Gamma_{2} /\left(\Gamma_{1}+\Gamma_{2}\right)^{2}$. Rather than four peaks, we see three distinct plateaus when $h_{0}=0-G / G_{0}=1$ for the plateaus centered about $N_{0}=1$ and $N_{0}=3 ; G / G_{0}=2$ for the plateau centered about $N_{0}=2$. Furthermore, $h_{0}$ has interesting effects on $G$ - whereas $h_{0}$ mainly splits the peaks in the UV regime (Fig. 1 1 ), $h_{0}$ has more drastic effects in the IR. Indeed, the plateau centered about $N_{0}=2$ is suppressed by $h_{0}$; the plateaus centered about $N_{0}=1$ and $N_{0}=3$, on the other hand, are unaffected. As discussed below, the behavior at $T=0$ occurs because SC states between the QD and leads are formed; $h_{0}$ has drastic effects on the SC states.

We now address the physics behind Fig. 1 - we investigate the SC states which arise in the IR, as well as how they evolved from the UV FP. To this end, we examine the QD's spectral function (SF), $A_{d}(\omega)$. Fig. 2 shows $A_{d}(\omega)$ (at $\left.T=0\right)$ obtained via the LDECA. For comparison, results for $A_{d}(\omega)$ at the UV FP - obtained by formally setting $\left\{t_{i}\right\}=0$ - are shown in the insets. Fig. [2] shows $A_{d}(\omega)$ at the $N_{0}=1 / 2 \mathrm{CB}$ peak. Here we see a broad peak near $\omega=0$ i.e. near $E_{F}$; its features do not change much with $h_{0}$. From the insets, we see there was a redistribution of spectral weight, with much of the peak's weight in the IR having been transferred from higher energies.

Figs. 2 $\mathrm{b}$ and $2 \mathrm{r}$ show $A_{d}(\omega)$ in the CB valleys. A key feature is the narrow resonance which appears at or near $E_{F}$ - the Kondo resonance (KR). This resonance is a consequence of the SC state formed between the $\mathrm{QD}$ and
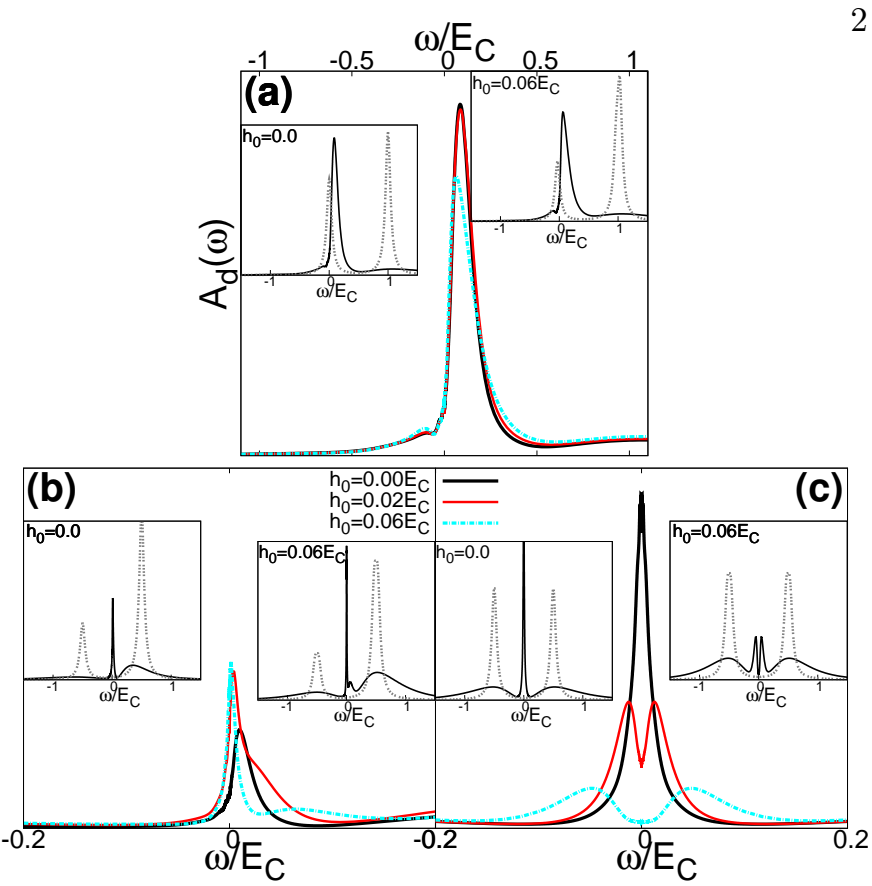

FIG. 2: QD's spectral function $A_{d}(\omega)$. (a) $A_{d}(\omega)$ for $N_{0}=1 / 2$. (b) $A_{d}(\omega)$ for $N_{0}=1$. (c) $A_{d}(\omega)$ for $N_{0}=2$. Insets: Comparison of $A_{d}(\omega)$ at the UV (gray dotted lines) and IR (solid black lines) fixed points.

leads due to the KE; its width represents the dynamically generated scale characteristic of the SC state the Kondo temperature, $T_{K}$. As discussed below, the position and width of the KR are characteristic of the particular Kondo fixed point (KFP).

Fig. 2b shows $A_{d}(\omega)$ in the $N_{0}=1$ valley i.e. the $1 / 4$ filled QD. For $h_{0}=0, A_{d}(\omega)$ exhibits a KR near $E_{F}$; for $h_{0} \neq 0$, the resonance moves toward $E_{F}$ and its width narrows. As mentioned above, when $h_{0}=0$ the system has an $\mathrm{SU}(4)$ symmetry; $h_{0} \neq 0$ reduces this symmetry to $\mathrm{SU}(2)$. For the 1/4-filled QD, the system flows to the SU(4) KFP when $h_{0}=0$, while $h_{0} \neq 0$ drives the system to the $\mathrm{SU}(2)$ $\mathrm{KFP}$; the KR is near (at) $E_{F}$ at the $\mathrm{SU}(4)(\mathrm{SU}(2)) \mathrm{KFP}$ with $T_{K}^{\mathrm{SU}(2)}<T_{K}^{\mathrm{SU}(4)}$. The UV and IR behaviors of $A_{d}(\omega)$ are compared in the insets - the KR is indeed an IR property, with its spectral weight taken from the higher energy UV peaks; interestingly, $h_{0}$ does not change the qualitative features of $A_{d}(\omega)$ at either the UV or IR FPs.

Fig. 2 r shows $A_{d}(\omega)$ for $N_{0}=2$ i.e. the $1 / 2$-filled QD its behavior is drastically different from the SFs arising for both $N_{0}=1 / 2$ and $N_{0}=1$. For $h_{0}=0, A_{d}(\omega)$ exhibits a narrow $\mathrm{KR}$ at $E_{F}$; for $h_{0} \neq 0$, the resonance splits and is suppressed. Hence, contrary to the $1 / 4$-filled QD where $h_{0}$ drives the system from the $\mathrm{SU}(4) \mathrm{KFP}$ to the $\mathrm{SU}(2)$ $\mathrm{KFP}, h_{0}$ destroys the KE for the $1 / 2$-filled QD. The UV and IR behaviors of $A_{d}(\omega)$ are compared in the insets we see the KR suppressed as $h_{0}$ increases; as this occurs, the peaks at $\omega= \pm E_{C} / 2$ regain spectral weight.

Having discussed the QD's SF in the various regimes, we now discuss (further) consequences of the SF's features in the CB valleys i.e. for $N_{0} \simeq N$. To facilitate the analysis, we integrate out charge fluctuations on the QD; 
we arrive at the Coqblin-Schrieffer Hamiltonian [18]

$$
H_{\mathrm{QD}}=-\frac{J}{4}\left(\psi_{\kappa s}^{\dagger} f_{\kappa s}\right)\left(f_{\kappa^{\prime} s^{\prime}}^{\dagger} \psi_{\kappa^{\prime} s^{\prime}}\right)-\frac{h_{0}}{2} f_{\kappa s}^{\dagger} \sigma_{\kappa \kappa^{\prime}}^{z} f_{\kappa^{\prime} s}
$$

where $\psi_{\kappa s}=\left[t_{1} \psi_{1 \kappa s}(0)+t_{2} \psi_{2 \kappa s}(0)\right] / t$ with $t=\sqrt{t_{1}^{2}+t_{2}^{2}}$, $J=\left(4 t^{2} / E_{C}\right)\left[\left(N-N_{0}-1 / 2\right)^{-1}-\left(N-N_{0}+1 / 2\right)^{-1}\right]$, and the fermion operators satisfy the constraint $f_{\kappa s}^{\dagger} f_{\kappa s}=N$ with $N$ being the number of particles on the $\mathrm{QD}$. (While discussing the physics of the $\mathrm{CB}$ valleys, we write the QD's fermion operators as $\left\{f_{\kappa s}\right\}$; also, Einstein summation convention is utilized). To treat Eq. 3, we consider a path integral representation of the partition function - we enforce the constraint $f_{\kappa s}^{\dagger} f_{\kappa s}=N$ with a Lagrange multiplier field $\lambda$; we decouple the Kondo interaction using a Hubbard-Stratonovich field $\chi$. [18] We arrive at an effective Hamiltonian

$$
\begin{aligned}
H_{\mathrm{eff}} & =-\frac{h_{0}}{2} f_{\kappa s}^{\dagger} \sigma_{\kappa \kappa^{\prime}}^{z} f_{\kappa^{\prime} s}+\lambda\left(f_{\kappa s}^{\dagger} f_{\kappa s}-N\right) \\
& +\frac{4}{J}|\chi|^{2}+\chi^{\dagger} f_{\kappa s}^{\dagger} \psi_{\kappa s}+\chi \psi_{\kappa s}^{\dagger} f_{\kappa s} .
\end{aligned}
$$

We begin by considering the physics at higher energies, focussing on the flow from the UV to the IR FPs. To do so, we treat the Bose fields $\chi$ and $\lambda$ in Eq. 4 in mean-field theory (MFT). Treating $\lambda$ in MFT amounts to treating the constraint $f_{\kappa s}^{\dagger} f_{\kappa s}=N$ on average: $\left\langle f_{\kappa s}^{\dagger} f_{\kappa s}\right\rangle=N$. To describe the physics near the UV FP, we take $\langle\chi\rangle=0$; the physics of the KE is contained in the effective action for $\chi$, obtained by integrating out the $f$-fermions and leads. To one-loop order, the propagator of the $\chi$ field $\mathcal{J}\left(i \omega_{m}\right)$ is given by the diagram in Fig. 3 (with $\omega_{m}$ being a boson Matsubara frequency). Physically, $\mathcal{J}\left(i \omega_{m}\right)$ is the running Kondo coupling. 20]

Using our result for $\mathcal{J}\left(i \omega_{m}\right)$, the current $I=\langle\hat{I}\rangle$ was computed as per Ref. 16; results for $G / G_{0}$ vs. $T / T_{K}^{\mathrm{SU}(4)}$ are shown in Fig. 4 where

$$
T_{K}^{\mathrm{SU}(4)}=D \exp \left(-1 / \rho_{0} J\right)
$$

with $D$ being half the leads' bandwidth. [As before, $G_{0}=\left(e^{2} / \pi\right) 4 \Gamma_{1} \Gamma_{2} /\left(\Gamma_{1}+\Gamma_{2}\right)^{2}$.] Fig. 4a shows results for the 1/4-filled QD. To begin with, we see that $G$ grows logarithmically as $T$ is reduced - this is a consequence of the logarithmic growth of the running Kondo coupling. [18] Furthermore, $G / G_{0}$ always grows to $\mathcal{O}(1)$ i.e. the system always flows to strong coupling. This is because the 1/4-filled QD exhibits a $\mathrm{KE}$, irrespective of the value of $h_{0}$. However, $G$ grows more slowly for larger $h_{0}$ - the system flows to the $\mathrm{SU}(4)$ (SU(2)) KFP for smaller (larger) $h_{0}$; the slower growth of $G$ for larger $h_{0}$ occurs because $T_{K}^{\mathrm{SU}(2)}<T_{K}^{\mathrm{SU}(4)}$. (See Fig. 2b.)

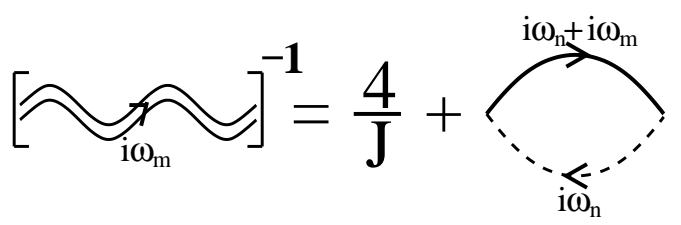

FIG. 3: $\chi$ field propagator $\mathcal{J}\left(i \omega_{m}\right)$ - the solid (dashed) line denotes the leads' ( $f$-fermions') Green's function.

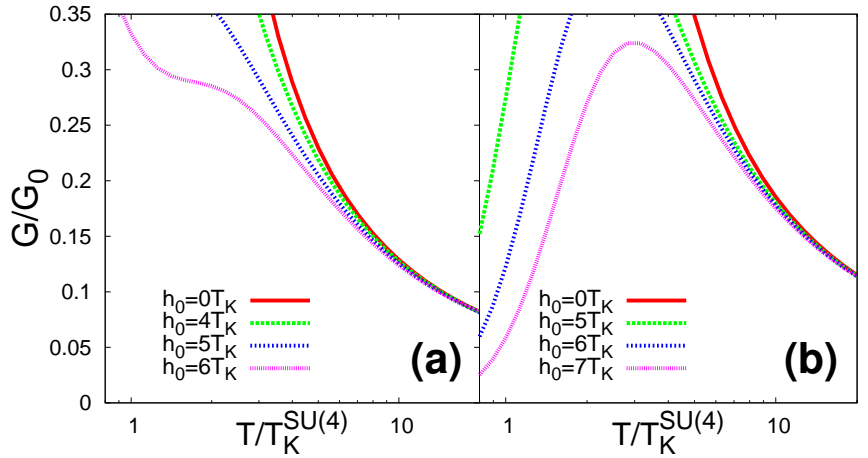

FIG. 4: $G / G_{0}$ vs. $T / T_{K}^{\mathrm{SU}(4)}$ in linear response in the $\mathrm{CB}$ valleys: (a) 1/4-filled QD and (b) 1/2-filled QD.

Fig. 40 shows $G / G_{0}$ vs. $T / T_{K}^{\mathrm{SU}(4)}$ for the $1 / 2$-filled $\mathrm{QD}$; the results are drastically different from the $1 / 4$ filled QD. As discussed above, whereas $h_{0}$ drives the system from the $\mathrm{SU}(4)$ to the $\mathrm{SU}(2) \mathrm{KFP}$ for the $1 / 4$ filled QD, $h_{0}$ destroys the KE for the $1 / 2$-filled QD (see Fig. 2re); $G$ even becomes non-monotonic. Such behavior has been observed in magnetic alloys, where the occurrence of a spin glass phase freezes spin-flip processes and, hence, suppresses the KE. 21] Here, larger values of $h_{0}$ freeze both spin and orbital processes. More precisely, $h_{0}$ cuts off the growth of the running Kondo coupling for the $1 / 2$-filled QD $\mathcal{J}(T) \equiv \mathcal{J}\left(i \omega_{m}=0\right)$ is given by

$$
\rho_{0} \mathcal{J}(T)=J\left\{\ln \left(\frac{2 \pi T}{T_{K}^{\mathrm{SU}(4)}}\right)+\operatorname{Re}\left[\psi\left(\frac{1}{2}+i \frac{h_{0}}{4 \pi T}\right)\right]\right\}^{-1},
$$

where $\psi(z)$ is the digamma function; [22] as $\psi(z) \simeq \ln (z)$ for $|z| \gg 1$, the growth of $\mathcal{J}(T)$ is suppressed for $h_{0}$ sufficiently larger than $T_{K}^{\mathrm{SU}(4)}$.

Having discussed the flow (in the $\mathrm{CB}$ valleys) from the UV to the KFPs in the IR, we now discuss further the physics of the SC KFPs. As before, we treat the Bose fields in Eq. 4 in MFT. Now, however, to describe the physics of the SC KFPs, we take $\langle\chi\rangle=\chi_{0}(\neq 0)$. 18] Hence, $\lambda$ and $\chi_{0}$ are determined via $\left\langle f_{\kappa s}^{\dagger} f_{\kappa s}\right\rangle=N$ and $\chi_{0}+2 J\left\langle\psi_{\kappa s}^{\dagger} f_{\kappa s}\right\rangle=0$. With $\langle\chi\rangle \neq 0$, the $f$-fermions SF is

$$
A_{i}^{f}(\omega)=\frac{2 \Gamma}{\left(\omega-\varepsilon_{i}\right)^{2}+\Gamma^{2}}
$$

$\left(\varepsilon_{1 / 2}=\lambda \pm h_{0} / 2\right)$, where $\Gamma=T_{K}$ when $T=0$. [18]

Fig. [5] shows $G / G_{0}$ vs. $h_{0} / T_{K}^{\mathrm{SU}(4)}$ (computed as per Ref. 16) at $T=0$. For the $1 / 4$-filled $\mathrm{QD}, G / G_{0}=1$ regardless of the value of $h_{0}$. This occurs because there is always a KE, $\Gamma \neq 0$ - for small $h_{0}$, one is in the SU(4) Kondo regime; for larger $h_{0}$, one crosses over to the $\mathrm{SU}(2)$ Kondo regime. For the $1 / 2$-filled $\mathrm{QD}$, on the other hand, we see that $G$ depends on the magnitude of $h_{0}-G / G_{0}=2$ for $h_{0}=0$ and decreases as $h_{0}$ is increased. [Within MFT, $G \rightarrow 0$ for $h_{0}=2 T_{K}^{\mathrm{SU}(4)}$.] This is because the $\mathrm{SU}(4) \mathrm{KE}$ is destroyed and, consequently, the KR in the QD's SF is suppressed for $h_{0}$ sufficiently large. (See Fig. 2r.)

Also shown in Fig. [5]are results for the noise (which has been shown to be a powerful probe of Kondo physics 23, 


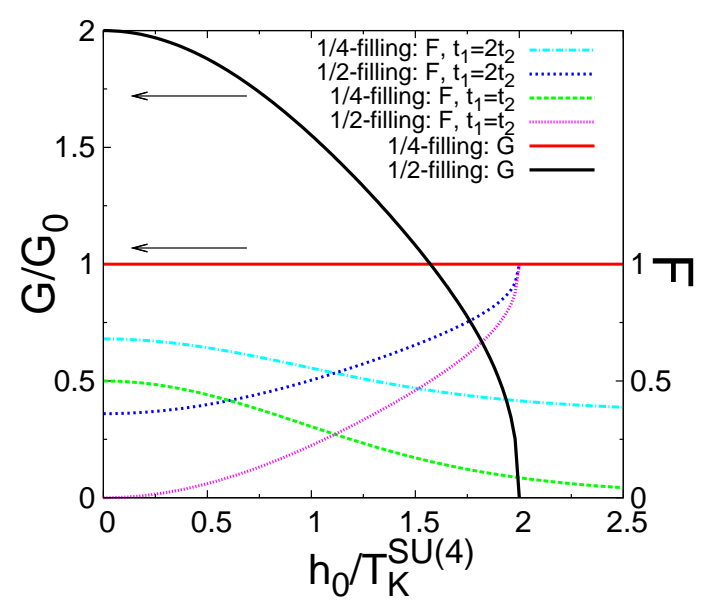

FIG. 5: $G / G_{0}$ vs. $h_{0} / T_{K}^{\mathrm{SU}(4)}$ and $F$ vs. $h_{0} / T_{K}^{\mathrm{SU}(4)}$ in linear response at $T=0$.

24]) at $T=0$. More specifically, we computed the zerofrequency noise

$$
S(e V)=\int d t\left[\langle\hat{I}(t) \hat{I}\rangle-\langle\hat{I}\rangle^{2}\right]
$$

and, subsequently, the Fano factor $F \equiv S / 2 e I$ in linear response. While the conductance probes the spectral weight of the KR, the noise gives information about its position. Indeed, while both the $\mathrm{SU}(4)$ and $\mathrm{SU}(2)$ KEs give $G / G_{0}=1$ for the $1 / 4$-filled $\mathrm{QD}$, differences between the two can drastically be seen in $F-F$ decreases as $h_{0}$ increases i.e. as we move from the $\mathrm{SU}(4)$ to the $\mathrm{SU}(2)$ KFP. This is seen most dramatically when $t_{1}=t_{2}$ where $F \rightarrow 0$ as $h_{0}$ increases, but the qualitative behavior of $F$ is robust. [See the results for $t_{1}=2 t_{2}$.] Physically, this arises because the KR in the QD's $\mathrm{SF}$ is at $E_{F}$ at the $\mathrm{SU}(2) \mathrm{KFP}$, while it is away from $E_{F}$ at the $\mathrm{SU}(4) \mathrm{KFP}$. (See Fig. 2b.) For the $1 / 2$-filled QD, $F$ increases as $h_{0}$ increases, approaching unity as $G \rightarrow 0$; for $t_{1}=t_{2}, F \rightarrow 0$ as $h_{0} \rightarrow 0$. For $h_{0}=0$, the system is in a SC state, with a KR at $E_{F}$; as a result $F=0$ when $t_{1}=t_{2}$. As $h_{0}$ is increased, the SC state is destroyed and the system is driven to the weak-coupling regime; hence, $F \rightarrow 1$ i.e. $F$ becomes Poissonian. 25]

To summarize, we considered the behavior of CNT QDs in a magnetic field. We analyzed the evolution of the system from the UV to the IR FPs. We discussed the KEs that occur and their experimental consequences. In particular, the KEs arising for the 1/4-filled and 1/2filled QDs were shown to be drastically different, with distinct signatures in the system's transport; we are optimistic our results, particularly for the noise, 26] can be observed experimentally. Besides CNT QDs, our results are relevant to double QDs and, more generally, to QDs with two-fold orbital degeneracy.

This work was supported by the NSERC of Canada (MM and EHK), a SHARCNET Research Chair (MM and EHK), and the NSF (DMR - 0710529) (GBM).
[1] For reviews, see C. Dekker, Physics Today 52(5), 22 (1999); Physics World 13(6) (2000); R. Saito, G. Dresselhaus, and M. S. Dresselhaus, Physical Properties of Carbon Nanotubes (Imperial College Press, London 1998).

[2] S. J. Tans, M. H. Deverot, H. Dai, A. Thess, R. E. Smalley, L. J. Geerligs, and C. Dekker, Nature (London) 386, 474 (1997); Z. Yao, H. W. Ch. Postma, L. Balents, and C. Dekker, Nature (London) 402, 273 (1999).

[3] H. W. Ch. Postma, T. Teepen, Z. Yao, M. Grifoni, and C. Dekker, Science 293, 76 (2001).

[4] C. Zhou, J. Kong, E. Yenilmez, and H. Dai, Science 290, 1552 (2000).

[5] M. Bockrath, D. H. Cobden, P. L. McEuen, N. G. Chopra, A. Zettl, A. Thess, and R. E. Smalley, Science 275, 1922 (1997); J. Kong, C. Zhou, E. Yenilmez, and H. Dai, Appl. Phys. Lett. 77, 3977 (2000); M. J. Biercuk, S. Garaj, N. Mason, J. M. Chow, and C. M. Marcus, Nano Lett. 5, 1267 (2005).

[6] J. Nygard, D. H. Cobden, and P. E. Lindelof, Nature 480, 342 (2000).

[7] For a review, see, H. Grabert and M. H. Devoret, Eds., Single Charge Tunneling (Plenum, New York 1991).

[8] L. Kouwenhoven and L. Glazman, Physics World 14(1), 33 (2001).

[9] P. Jarillo-Herrero, J. Kong, H. S. J. van der Zant, C. Dekker, L. P. Kouwenhoven, and S. DeFranceschi, Nature 434, 484 (2005).

[10] A. Makarovski, A. Zhukov, J. Liu, and G. Finkelstein, Phys. Rev. B 75, 241407(R) (2007).

[11] A. Makarovski, J. Liu, and G. Finkelstein, Phys. Rev.
Lett. 99, 066801 (2007).

[12] M. S. Choi, R. Lopez, and R. Aguado, Phys. Rev. Lett. 95, 067204 (2005); J. S. Lim, M.-S. Choi, M. Y. Choi, R. Lopez, and R. Aguado, Phys. Rev. B 74, 205119 (2006).

[13] E. Minot, Y. Yaish, V. Sazonova, and P. L. McEuen, Nature 428, 536 (2004).

[14] F. B. Anders, D. E. Logan, M. R. Galpin, and G. Finkelstein, Phys. Rev. Lett. 100, 086809 (2008).

[15] For a review of $\mathrm{SU}(4) \mathrm{KEs}$ in nanostructures, see G. Zarand, Philos. Mag. 86, 2043 (2006).

[16] Y. Meir and N. Wingreen, Phys. Rev. Lett. 68, 2512 (1992).

[17] E. V. Anda, G. Chiappe, C. A. Büsser, M. A. Davidovich, G. B. Martins, F. Heidrich-Meisner, and E. Dagotto, Phys. Rev. B 70, 085308 (2008).

[18] A. Hewson, The Kondo Effect to Heavy Fermions (Cambridge University Press, Cambridge 1993).

[19] C. W. J. Beenakker, Phys. Rev. B 44, 1646 (1991).

[20] P. Coleman, Phys. Rev. B 35, 5072 (1987).

[21] See, e.g. F. Schopfer, C. Bäuerle, W. Rabaud, and L. Saminadayar, Phys. Rev. Lett. 90, 056801 (2003).

[22] I. S. Gradshteyn and I. M. Ryzhik, Table of Integrals, Series, and Products (Academic Press, San Diego 1994).

[23] Y. Meir and A. Golub, Phys. Rev. Lett. 88, 116802 (2002); E. Sela, Y. Oreg, F. von Oppen, and J. Koch, Phys. Rev. Lett. 97, 086601 (2006).

[24] T. Delattre, C. Feuillet-Palma, L. G. Herrmann, P. Morfin, J.-M. Berroir, G. Feve, B. Placais, D. C. Glattli, M.-S. Choi, C. Mora, and T. Kontos, Nature Physics 5, 208 (2009). 
[25] Ya. M. Blanter and M. Büttiker, Phys. Rep. 336, 1 (2000).

[26] Indeed, by working at lower temperatures and in a mag- netic field, we are optimistic our results for the noise could be observed in the device/setup of Ref. 24 . 